\title{
Prevalence of Tonsillar Actinomycosis in Tonsillectomy Specimens; Clinical Importance and Management
}

\author{
(1) Naeimeh Daneshmandan1', (1) Mohammadebrahim Yarmohammadi2, (1) Poopak Izadi2 \\ 1University of Social Welfare and Rehabilitation Sciences, Department of Pediatric Neurorehabilitation Research Center, Tehran, Iran \\ 2Shahed University, Department of Otorhinolaryngology, Tehran, Iran
}

\begin{abstract}
Aim: We aimed to assess the prevalence of actinomycosis in tonsillectomy specimens and its clinical importance and management in our center.

Materials and Methods: In this retrospective matched case-control study, patients who underwent tonsillectomy during $2010-2015$ in Shahid Mostafa Khomeini Hospital, Tehran, were studied. Data regarding age, sex, indication for surgery (such as recurrent tonsillitis or sleepdisordered breathing), tonsil size, and length of disease before surgery were collected in both histopathologically positive (case) and negative (control) patients for actinomycosis.

Results: From the 260 studied tonsillectomy specimens, actinomycosis was found in 20 (8.75\%) and 60 Actinomycosis negative cases were chosen randomly for the control group. There was no statistically significant difference between the groups with respect to age, sex, length of disease before surgery, and incidence of actinomycosis in tonsillectomy due to recurrent tonsillitis or sleep-disordered breathing. However, the tonsil size was significantly greater in specimens positive for actinomycosis $(8.65 \pm 1.5 \mathrm{~mL}$ versus $4.38 \pm 0.22 \mathrm{~mL}, p<0.001)$.

Conclusion: Actinomycosis in tonsillectomy specimens is an incidental histopathological finding and does not necessarily correlate with active disease; while it is associated with tonsillar hypertrophy and increased tonsil size.
\end{abstract}

Keywords: Actinomycosis, tonsillectomy, recurrent tonsillitis, tonsillar hypertrophy

\section{Introduction}

Tonsillar Actinomycosis is a rare pathological condition in tonsillectomy specimens and its clinical importance is still controversial (1). Tonsillectomy is the most frequent surgery in children (2). The most common indications for tonsillectomy are recurrent tonsillitis and obstructive diseases such as sleep apnea, snoring, and mouth breathing
(3). The histopathological examination of tonsillectomy specimens is done routinely to rule out occult malignancy or granulomatous disease (2-4).

The prevalence of Actinomyces in tonsil specimens varies from $1.3-37 \%$ (5-7). Actinomyces are a genus of grampositive, pleomorphic, non-spore forming anaerobic, nonacid-fast branched filamentous bacteria (7). Six species of Actinomyces may cause disease in humans, but the most 
common isolated species are Actinomyces israelii and Actinomyces naeslundii (7).

The pathogenic Actinomyces species are commensals and saprophytic flora of the oropharynx, gastrointestinal tract and female genital tract and humans are a natural reservoir of pathogenic Actinomyces species (8).

Actinomycosis is a chronic disease which can form abscess, tissue fibrosis and secretory sinuses (9). The most common type for Actinomycosis is cervicofacial with a prevalence of $11-97 \%$ (10). The route of entry of the microorganism into the gastrointestinal tract from the mouth to rectum, is typically a break or trauma in the mucosa. Integrity of mucosal membrane is an important factor against infections. But this mechanical barrier is very thin and fragile in the gingival margin and tonsillar crypts and mucosal trauma in the tonsillar surface leads to a proliferation of these fastidious anaerobic bacteria in tonsillar crypts. Actinomyces species normally colonize tonsillar crypts and the oral cavity and many Actinomyces infections have odontogenic origins $(9,10)$.

The prevalence of tonsillar Actinomycosis has been reported as higher in patients with sickle cell anemia, beta $(\beta)$ thalassemia and $\beta$-hemolytic streptococcus (7). These conditions diminish the oxidation-reduction potential and cause a proliferation of Actinomyces in tonsillar tissue. This process may lead to tonsillar hypertrophy (7).

It has been suggested that tonsillar Actinomycosis may be an etiological factor in obstructive tonsillar hypertrophy and chronic tonsillitis $(5,7,11-14)$. We aimed to assess the relationship between Actinomycosis in tonsillectomy specimens and the main causes of tonsillectomy as recurrent tonsillitis or sleep disordered breathing to calculate its prevalence in our center.

\section{Materials and Methods}

In this matched case-control study we gathered data from Actinomycosis histopathology samples of 20 patients who underwent tonsillectomy during 2010-2015 in Shahid Mostafa Khomeini Hospital, Tehran, Iran. To increase study power, we chose 60 Actinomycosis negative (An) tonsillectomy specimens for the control group. Power analysis shows that sample size was enough to assess associations.

Epidemiological data including age, sex, indication for tonsillectomy such as recurrent tonsillitis or sleep disordered breathing, tonsil size, and length of disease before surgery were recorded in both the Actinomycosis positive (Ap) and An groups.

Sleep disordered breathing was defined by snoring and mouth breathing during sleep or an irregular sleep pattern with clinically significant awakening from sleep with choking or gasping. Recurrent tonsillitis was diagnosed as greater than 6 acute tonsillitis attacks in 1 year or 3 in 2 consecutive years $(2,3)$.

Tonsillectomy was performed with the cold dissection and suture ligation method and tonsils were sent in formalin to the pathology department. Histopathological analysis of the specimens was performed by two pathologists in the pathology ward of the hospital using direct microscopy and hematoxylin-eosin staining which is highly effective in showing Actinomyces colonies. Mean tonsillar size was recorded as the multiplication of length by height by width of tonsils measured and recorded in the pathologic results. The assessment of each tissue sample included an evaluation of mucosal surface, crypts and associated lymphoid tissue. The pathological feature of Actinomycosis was explained as aggregates of filamentous basophilic microorganisms arranged in radial spoke-like fusions. The diagnosis of Actinomycosis is established by two of the following conditions: positive culture, sulfur granules, and biopsy specimens showing the organisms (15).

Sulfur granules in infections other than Actinomycosis are very rare; therefore, their presence strongly supports the diagnosis (Figure 1). Although the ideal route for diagnosis is culture, its failure rate is very high because of the overgrowth of other microorganisms, lack of growth due to previous antibiotic therapy and inadequate anaerobic conditions or short-term incubation (16). Therefore, histopathological features from surgical specimens is the most preferred tool for diagnosis (Figures 2 and 3). Because our research was retrograde and on the pathologic specimens, and all the datas were without any information about patients, research was done completely secret and there was no need to ethics committee approval and informed consent.

\section{Statistical Analysis}

Logistic regression analysis was used to assess the relationship between tonsil size and Actinomycosis, after controlling confounding variables. Data were analyzed using SPSS software, version 16.

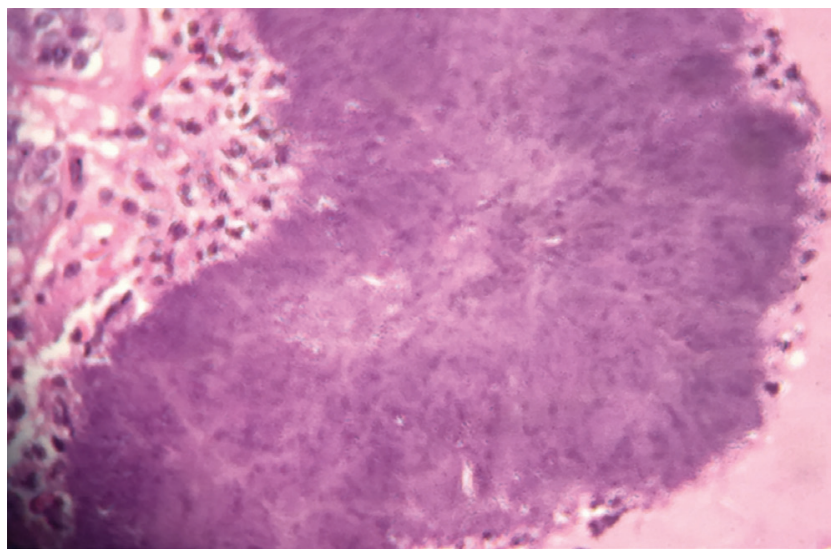

Figure 1. Sulfur granule 
The categorical variables were analyzed with chisquare test and non-parametric data with Mann-Whitney U test.

Independent Variables: Data regarding age, sex, indication for surgery (such as recurrent tonsillitis or sleepdisordered breathing), tonsil size, and length of disease before surgery were collected.

Dependent Variables: Histopathologically positive (case) and negative (control) patients for actinomycosis.

Confounding Variables: Sex, indication for surgery (such as recurrent tonsillitis or sleep-disordered breathing)

Main Variable: Tonsil size.

\section{Results}

Twenty (8.75\%) specimens (out of 260) were positive for Actinomycosis. Sixty (An) patients were chosen for

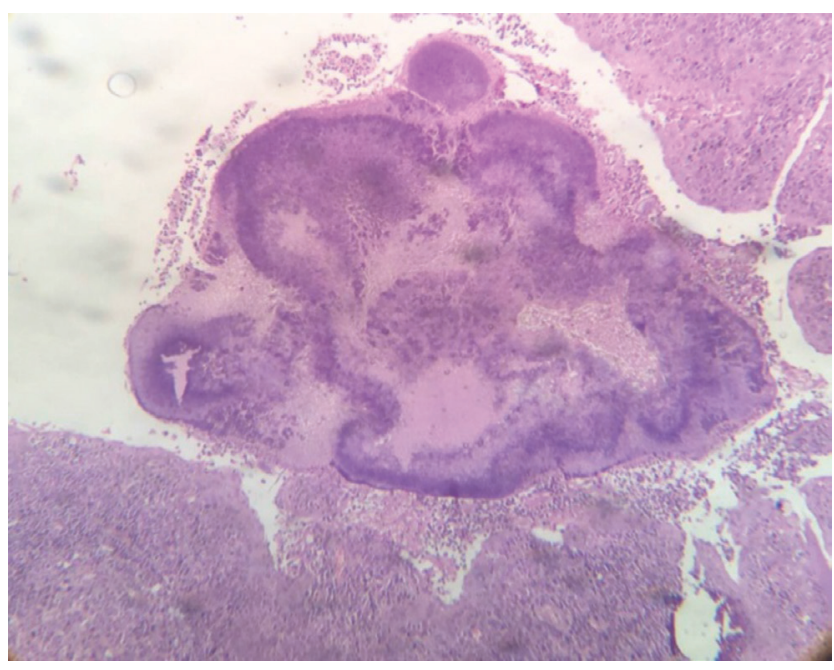

Figure 2. Actinomycosis clump formation

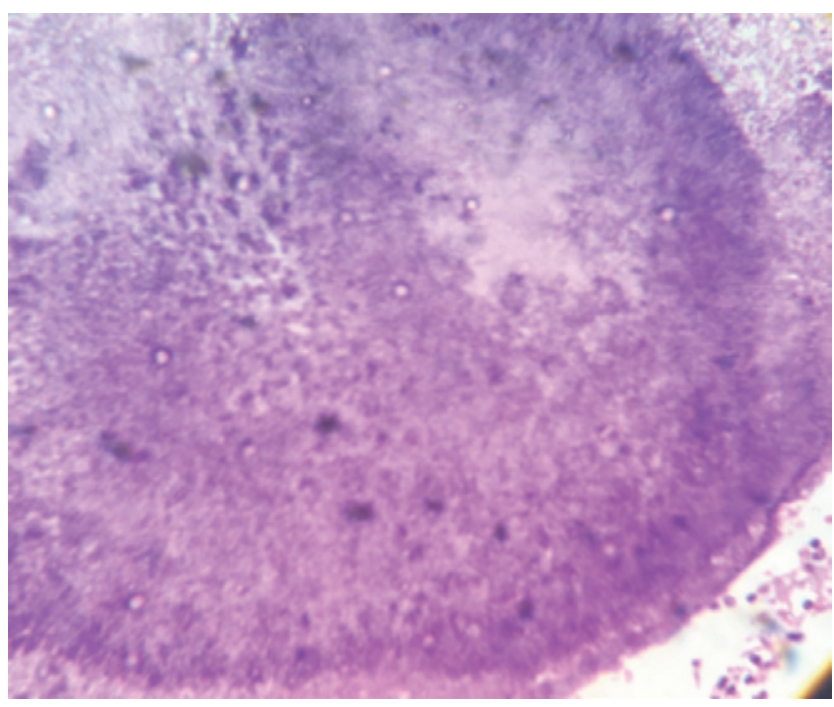

Figure 3. Aggregates of filamentous basophilic microorganisms in Actinomycosis the control group. The patients' demographic data are summarized in Table I.

The mean \pm standard deviation (SD) ages of patients in the Ap and An groups were 16.24 44.73 (range: $4-24$ years) and 12.02 \pm 2.07 (range: $3-20)$ years, respectively $(p=0.124)$. There were 9 (42.9\%) women and 11 (57.1\%) men in the Ap group and 26 (43.3\%) women and 34 (56.7\%) in the An group ( $p=0.927)$.

All the patients in the case group had recurrent tonsillitis and $14(66.7 \%)$ had sleep disordered breathing as the indication for surgery. In the An group, 58 (98.3\%) patients had recurrent tonsillitis and 31 (51.7\%) had sleep disordered breathing. There was no statistically significant difference between the two groups in recurrent tonsillitis $(p=1)$ or sleep disordered breathing $(p=0.234)$.

Mean \pm SD length of disease before surgery was $6.2 \pm 1$ year in the Ap group and 5.8 \pm 1 year in the An group ( $p>0.05$ ). The mean \pm SD tonsil size in the Ap group was $8.65 \pm 1.54 \mathrm{~mL}$ and in the An group was $4.38 \pm 0.22 \mathrm{~mL}(p<0.001)$. Logistic regression analysis showed that Actinomycosis increased the tonsil size bz 1.8 times (OR: 1.8, Cl: 95\%, 1.2-4.3).

\section{Discussion}

Prevalence: In our study the prevalence of tonsillar actinomycosis was $8.75 \%$. The prevalence of Actinomycosis in the tonsils varies from $1.3 \%$ to $37 \%(5-7)$ and it has been identified in $6.7 \%$ (17), $18.3 \%$ (5), 29.5\% (20), 28.5\% (7), and $11.5 \%$ (18) of all tonsil specimens in different studies. The wide range of variation in reported prequencies have been attributed to different staining techniques by different laboratories and a difference in patients and working groups as well as indications for tonsillectomy $(17,18)$.

Age and Sex: Some studies reported a relationship between Actinomyces colonization and age (17-19). Ashraf and colleagues reported a mean age of 23.34 years for patients with actinomycosis and 15.91 years for those without actinomycosis indicating a positive correlation with older

\begin{tabular}{|l|l|l|l|}
\hline \multicolumn{4}{|l|}{$\begin{array}{l}\text { Table I. Demographic data of the patients in the case and } \\
\text { control groups }\end{array}$} \\
\hline $\begin{array}{l}\text { Demographic } \\
\text { data }\end{array}$ & $\begin{array}{l}\text { Actinomycosis } \\
\text { positive }\end{array}$ & $\begin{array}{l}\text { Actinomycosis } \\
\text { negative }\end{array}$ & $\begin{array}{l}\text { p } \\
\text { value }\end{array}$ \\
\hline Mean age (years) & $16.24 \pm 4.73$ & $12.02 \pm 2.07$ & $>0.05$ \\
\hline Sex & $\begin{array}{l}9 \text { female, } 11 \\
\text { male }\end{array}$ & $\begin{array}{l}26 \text { female, } 34 \\
\text { male }\end{array}$ & $>0.05$ \\
\hline Recurrent tonsillitis & $20(100 \%)$ & $58(98.3 \%)$ & $>0.05$ \\
\hline $\begin{array}{l}\text { Sleep disordered } \\
\text { breathing }\end{array}$ & $14(66.7 \%)$ & $31(51.7 \%)$ & $>0.05$ \\
\hline $\begin{array}{l}\text { Duration of disease } \\
\text { before surgery } \\
\text { (years) }\end{array}$ & $6.2 \pm 1$ & $5.8 \pm 1$ & $>0.05$ \\
\hline Tonsil size $(\mathrm{mL})$ & $8.65 \pm 1.54$ & $4.38 \pm 0.22$ & $<0.001$ \\
\hline
\end{tabular}


age (19). Aydin et al. (17) and van Lierop et al. (18) and Toh et al. (6) and colleagues found that actinomycosis was more prevalent in adults than children. Some authors reported a female predominance of tonsillar Actinomycosis $(1,17)$, while one study did not show such a predominance (20). We found no correlation between tonsillar actinomycosis and sex or age because of the low number of adults in the two groups.

Clinical Relationship: Several studies have aimed to identify the relationship between tonsillar Actinomycosis and clinical tonsillar disease including recurrent tonsillitis or sleep-disordered breathing $(5,7,17,20,21)$.

This theory was suggested in 1910 by Lord stating that isolation of Actinomyces from tonsillar crypts may correlate with tonsillar hypertrophy due to the production of toxin by the microorganism (22). However, this has never been proven.

A higher prevalence of Actinomycosis was reported in specimens from tonsillectomy for obstructive symptoms compared with recurrent tonsillitis $(5-7,14,23)$. They suggested that tissue colonization with Actinomyces may have an etiological role in lymphoid hyperplasia and tonsillar hypertrophy $(5,7,14)$. Therefore, administration of a 12-week course of oral penicillin to all patients with obstructive symptoms has been suggested (5). However, it has not been proven by other studies $(4,7,20,21)$. Toh et al. (6) demonstrated that Actinomycosis does not have any effect on tonsillar size while another study (23) reported larger sizes of tonsils with Actinomycosis. In our study, tonsillar size was significantly greater in the case group. Therefore, colonization of microorganisms may have an important role in size.

One study showed that the prevalence of Actinomyces colonization was higher in patients with recurrent tonsillitis than sleep-disordered breathing, but no statistically significant correlation was found between the presence of Actinomycosis and tonsillar hypertrophy or recurrent tonsillitis (19).

Another study found no relationship between clinical tonsillar disease and the presence of Actinomyces, in spite of finding a higher rate of cryptitis in tonsils with Actinomycosis, histopathologically (17).

A strong correlation was found between Actinomycosis and chronic tonsillitis showing positive tissue reaction to Actinomyces colonization in tonsil crypts suggesting its probable role in chronic and antibiotic resistant tonsillar symptoms (1).

In two other studies, no tissue reaction was found due to Actinomyces colonization in tonsils, therefore they reported no correlation between tonsillar Actinomycosis and recurrent tonsillitis $(6,18)$. We also found no statistically significant difference in the indication for surgery such as recurrent tonsillitis or sleep disordered breathing between the two groups.
According to different studies it seems that Actinomyces colonization in tonsillar tissue does not indicate any active disease and there seems to be no correlation between its colonization and clinical tonsillar disease.

Histopathology: Tonsillar actinomycosis in the present study was diagnosed via the presence of sulfur granules in histopathological sectioning and confirmed by histopathologic analysis of specimens, but there was no specific tissue reaction such as cryptitis or micro-abscess formation or fibrosis in the specimens. The diagnosis of Actinomycosis was accurately done by the isolation of Actinomyces species from specimen culture or immunofluorescence staining, but less than $50 \%$ of cases were positive because of previous treatment with antibiotics, unsuitable culture media, or growth of other bacteria species $(24,25)$. The presence of Actinomycotic sulfur granules in histological sections is highly supportive for diagnosis because these granules are very rare in other infections, however it is not diagnostic (26).

In one study, microorganisms were situated deeply in tonsillar crypts in histological examination but there was no specific tissue reaction to Actinomyces colonization (18). Gaffney et al. (20) found no relationship between the presence of Actinomycosis and tonsillar fibrosis or microabscess in reviewing the histology results of tonsil specimens with Actinomycosis. Aydin et al. (17) found cryptitis in tonsillar tissue and suggested that cryptitis can be used as a histological indicator of tonsillar Actinomycosis.

Riffat and Walker (27) used histopathological analysis with hematoxylin-eosin staining plus microbiological culture for Actinomycosis and suggested that Actinomyces colonization in tonsillar tissue cannot be neglected as passive saprophytes and its correlation with clinical diseases must be further studied.

Management: Actinomyces species are susceptible to several antibiotics such as penicillin $G$, erythromycin, and tetracyclines (8). Penicillin $G$ is the drug of choice for all clinical forms of Actinomycosis and tetracycline is the drug of choice for patients who are allergic to penicillin $(24,28)$.

The treatment of choice for Actinomycosis in chronic fibrosing conditions is long-term high doses of antibiotics to penetrate into the suppuration and fibrosis, in conjunction with drainage of the abscess and surgical excision of fistula and sinus tracts (24).

In some trials, oral penicillin for 12 weeks was suggested as a medical management of tonsillar Actinomycosis $(5,29,30)$. However, in another study, this method appeared to be ineffective because, in some patients with sickle cell anemia who had received penicillin for a long period, there was Actinomycosis in their tonsils. Therefore, surgical excision has been recommended as the definitive treatment for head and neck Actinomycosis and tonsillectomy is the definite therapy for tonsillar Actinomycosis (7). 
We hypothesis that the presence of Actinomyces in tonsillar specimens may have an etiological role in tonsillar hypertrophy and long-term tonsillar problems and chronic tonsillitis refractory to antibiotics. However, there was no specific evidence of tissue reaction to Actinomyces in tonsillar specimens and their presence was found to be due to superficial colonization of the tonsillar crypts. Therefore, it seems to be an incidental finding.

\section{Study Limitations}

This study was based on data gathered from patient medical records. We were not able to investigate the relation between sleep disorders and nasal obstruction. It is probable that some patients with history of mouth breathing suffered from a very common disease such as allergic rhinitis, which was not identified in patients with sleep-disorder breathing. This nasal obstruction may also affect our data analysis and influence some associations between sleep-disorder breathing and tonsil size.

\section{Conclusion}

In this study, we found no relationship between tonsillar Actinomycosis and tonsillectomy indications such as recurrent tonsillitis and sleep disordered breathing. However, tonsil size in Ap specimens were found to be significantly larger than An ones. Therefore, the presence of Actinomyces colonization in tonsillar specimens may have an etiological role in tonsillar hypertrophy and long-term tonsillar lesions and chronic tonsillitis refractory to antibiotics with no correlation or questionable relation with tonsillectomy indications such as recurrent tonsillitis and sleep-disordered breathing. However, this needs further study with a larger number of specimens or long-term prospective studies with meticulous methods for identifying the microorganism.

\section{Acknowledgement}

We are grateful for the collaboration of Miss Ghamzoda and Dr Elham Faghihzadeh for contributing in the statistical analysis. We also thank Dr. Mehdi Noroozi, epidemiologist and Dr Mohammadreza Jalali Nodushan, Pathologist, for their sincere support in data analysis, interpretation and photography.

\section{Ethics}

Ethics Committee Approval: Because our research was retrograde and on the pathologic specimens, and all the datas were without any information about patients, research was done completely secret and there was no need to ethics committee approval.

Informed Consent: Because our research was retrograde and on the pathologic specimens, and all the datas were without any information about patients, research was done completely secret and there was no need to informed consent.
Peer-review: External and internal peer-reviewed.

\section{Authorship Contributions}

Surgical and Medical Practices: N.D., M.Y., P.I., Concept: P.I., Design: N.D., M.Y., P.I., Data Collection or Processing: N.D., M.Y., P.I., Analysis or Interpretation: N.D., M.Y., P.I., Literature Search: N.D., M.Y., P.I., Writing: N.D., M.Y., P.I.

Conflict of Interest: The authors declare that they have no conflict of interest.

Financial Disclosure: This research did not receive any specific grant from funding agencies in the public, commercial, or not-for-profit sectors.

\section{References}

1. Sujata N, Manimaran M, Rajeswara RN, Kafeel HA, Swayam IS. Histopathological features of tonsils and significance of actinomycosis in chronic tonsillitis. Journal of Dental and Medical Sciences 2015;14:105-9.

2. Sturm-O'Brien AK, Hicks JM, Giannoni CM, Sulek M, Friedman EM. Optimal utilization of histopathologic analysis of tonsil and adenoid specimens in the pediatric population. Int I Pediatr Otorhinolaryngol 2010;74:161-3.

3. Mohamad I, Hassan S, Salim R. The routine histopatlological examination of tonsillectomy specimens at hospital universiti sains malaysia-retrospective study and its implication. Malays I Med Sci 2007;14:19-21.

4. Mohamad I. Actinomyces in the tonsils: Hospital universiti sains malaysia experience. The Internet Journal of Otorhinolaryngology 2008;8:1-4.

5. Pransky SM, Feldman II, Kearns DB, Seid AB, Billman GF. Actinomycosis in obstructive tonsillar hypertrophy and recurrent tonsillitis. Arch Otolaryngol Head Neck Surg 1991;117:883-5.

6. Toh ST, Yuen HW, Goh YH. Actinomyces colonization of tonsils: a comparative study between patients with and without recurrent tonsillitis. J Laryngol Otol 2007;121:775-8.

7. Bhargava $D$, Bhusnurmath $B$, Sundaram $K R$, et al. Tonsillar actinomycosis: a clinicopathological study. Acta Trop 2001;80:163-8.

8. Raymond A, Smego JR, Foglia G. Actinomycosis. Clin Infect Dis 1998;26:1255-63.

9. Weese WC, Smith IM. A study of 57 cases of actinomycosis over a 36- year period. Arch Inter Med. 1975;135:1562-8.

10. Bennhoff D. Actinomycosis: diagnostic and therapeutic considerations and review of 32 cases. Laryngoscope 1984;94:1198-217.

11. Kutluhan A, Salvız M, Yalçıner G, Kandemir O, Yeşil C. The role of the actinomyces in obstructive tonsillar hypertrophy and recurrent tonsillitis in pediatric population. Int J Pediatr Otorhinolaryngol 2011;75:391-4.

12. Yasan H, Çiris M, Özel BF, Doğru H, Çandır O. The significance of histopathologic tonsillar actinomycosis in pediatric patients with recurrent acute tonsillitis. Elektronic Journal of Otolaryngoloy-Head and Neck Surgery 2006;5:1-4.

13. Priyadharshini SA, Subhashree AR, Ganaoathy H. Actinomycosis of tonsils-Incidental or Pathological? A case report. Int J Pharm Bio Scie 2014;5:164-8.

14. Ozgursoy OB, Kemal O, Saatci MR, Tulunay O. Actinomyces in the etiology of recurrent tonsillitis and obstructive tonsillar hypertrophy: answer from a histopathologic point of view. I Otolaryngol Head Neck Surg 2008;37:-865-9. 
15. Bennhoff DF. Actinomycosis: Diagnostic and therapeutic considerations and a review of 32 cases. Laryngoscope 1984;94:1198-217.

16. Maharjan S, Neopane P, Parajuli R. Actinomycosis with tonsillar diseases and its clinical significance. Global / Otolaryngol 2017;7:1-3.

17. Aydin A, Erkiliç S, Bayazit YA, Koçer NE, Ozer E, Kanlikama M. Relation between actinomycosis and histopathological and clinical features of the palatine tonsils: a comparative study between adult and pediatric patients. Rev Laryngol Otol Rhinol (Bord) 2005;126:95-8.

18. van Lierop AC, Prescott CA, Sinclair-Smith CC. An investigation of the significance of Actinomycosis in tonsil disease. Int I Pediatr Otorhinolaryngol 2007;71:1883-8.

19. Ashraf M), Azarpira N, Khademi B, Hashemi B, Shishegar. Relation between actinomycosis and histopathological and clinical features of the palatine tonsils: An Iranian Experience. Iran Red Crescent Med I 2011;13:499-502.

20. Gaffney R, Harrison M, Walsh M, Sweeney E, Cafferkey M. The incidence and role of actinomyces in recurrent acute tonsillitis. Clin Otolaryngol Allied Sci1993;18:268-71.

21. Melgarejo Moreno $P$, Hellin Meseguer $D$, Marco Garrido $A$, Galindo Ortego X, Ruiz Macia JA, Hostalet F. A correlation between age and actinomyces in the adenotonsillar tissue in children. B-ENT 2006;2:95-7.
22. Lord FT. The etiology of actinomycetes. The presence of actinomycetes in the contents of carious teeth and tonsillar crypts of patients with actinomycosis. IAMA 1910;55:1261-3.

23. San T, Gurkan E, Erdogan B, Ozkanl S. Does actinomyces have any role in tonsillar diseases children? İstanbul Med I 2014;15:209-12.

24. Mathew M. Diagnostic pathology of infectious diseases. Ear, nose, throat infections 2010:99-123.

25. Patnayak $R$, Jena $A$, Rukmangadha $N$, et al. Reactive actinomycotic tonsillar lesion. I Clin Res 2012;2:39-41.

26. Hotchi M, Schwartz J. Characterization of actinomycotic granules by architecture and staining methods. Arch Pathol 1972; $93: 392-400$

27. Riffat $F$, Walker P. Prevalence of tonsillar actinomyces in children undergoing tonsillectomy for sleep disordered breathing compared with recurrent tonsillitis. Int / Pediatr Otorhinolaryngol 2009;73:1111-3.

28. Smego RA. Actinomycosis. In: Hoeprich PD (ed). Infectious diseases. New York, J.B. Lippincott Company, 1994:493-7.

29. Maiwand O, Makey AR, Khagani A. Actinomycosis of the trachea affecting the right supraclavicular region. Thorax 1982;37:861-2.

30. Osborne JE, Blair RL, Christmas HE, Mckenzie H. Actinomycosis of the nasopharynx: a complication of nasal surgery. I Laryngol Otol 1988;102:639-40. 